\title{
NEW FUZZY DIFFERENTIAL SUBORDINATIONS
}

\author{
Georgia Irina OROS \\ Department of Mathematics, University of Oradea, Str. University, No.1 410087 Oradea, \\ ROMANIA
}

ABStraCt. In this paper, some new fuzzy differential subordinations obtained by using the integral operator $I_{\gamma}^{m}: A_{n} \rightarrow A_{n}$ introduced in 13 are obtained.

\section{INTRODUCTION AND PRELIMINARIES}

The notion of differential subordination was introduced by S.S. Miller and P.T. Mocanu in papers [6] and [7] and later developed in 8]. Many other authors have contributed to the development of this field of research. The notion of fuzzy subordination was recently introduced by G.I. Oros and Gh. Oros in paper 9 and the notion of fuzzy differential subordination was introduced by the same authors in 10. After that, some papers related to fuzzy differential subordinations have been published by the same authors, [11], 12], and by other authors, such as [3], 4] and [15].

Similar results on fuzzy differential subordinations obtained by using operators were recently published in [1], [2].

We next give the notations used throughout the paper:

Let $U$ denote the open disc in the complex plane, let $\bar{U}$ denote the closed unit disc in the complex plane and let $\partial U=\{z \in \mathbb{C}:|z|=1\}$. Let $\mathcal{H}(U)$ denote the class of analytic functions in the unit disc $U$.

We denote the following classes of analytic functions:

$$
A_{n}=\left\{f \in \mathcal{H}(U): f(z)=z+a_{n+1} z^{n+1}+a_{n+2} z^{n+2}+\ldots, z \in U\right\}
$$

with $A_{1}=A$;

$$
\mathcal{H}[a, n]=\left\{f \in \mathcal{H}(U): f(z)=a+a_{n} z^{n}+a_{n+1} z^{n+1}+\ldots, z \in U\right\}
$$

2020 Mathematics Subject Classification. 30C20, 30C45, 30A40.

Keywords and phrases. Analytic function, univalent function, fuzzy function, fuzzy differential subordination, dominant, best dominant.

घeorgia_oros_ro@yahoo.co.uk

(D) 0000-0003-2902-4455. 
for $a \in \mathbb{C}$ and $n \in \mathbb{N}^{*}$;

$$
S^{*}=\left\{f \in A: \operatorname{Re} \frac{z f^{\prime}(z)}{f(z)}>0, z \in U\right\},
$$

the class of starlike functions in $U$;

$$
C=\left\{f \in A: \exists g \in S^{*}, \operatorname{Re} \frac{z h^{\prime}(z)}{g(z)}>0, z \in U\right\}
$$

the class of close-to-convex (univalent) functions.

Definition 1. [8, Definition 2.2.b] We denote by $Q$ the set of functions $q$ that are analytic and injective on $\bar{U} \backslash E(q)$, where

$$
E(q)=\left\{\zeta \in \partial U: \lim _{z \rightarrow \zeta} q(z)=\infty\right\}
$$

and are such that $q^{\prime}(\zeta) \neq 0$ for $\zeta \in \partial U \backslash E(q)$. The set $E(q)$ is called exception set.

Lemma A. 8, Lemma 2.2.d] Let $q \in Q$ with $q(0)=a$ and let

$$
p(z)=a+a_{n} z^{n}+a_{n+1} z^{n+1}+\ldots
$$

be analytic in $U$, with $p(z) \neq a$ and $n \geq 1$. If $p$ is not subordinate to $q$, then there exist points $z_{0}=r_{0} e^{i \theta_{0}} \in U$ and $\zeta_{0} \in \partial U \backslash E(q)$, and an $m \geq n \geq 1$ for which $p\left(U_{r_{0}}\right) \subset q(U)$,

(i) $p\left(z_{0}\right)=q\left(\zeta_{0}\right)$,

(ii) $z_{0} p^{\prime}\left(z_{0}\right)=m \zeta_{0} q^{\prime}\left(\zeta_{0}\right)$ and

(iii) $\operatorname{Re}\left(\frac{z_{0} p^{\prime \prime}\left(z_{0}\right)}{p^{\prime}\left(z_{0}\right)}+1\right) \geq m \operatorname{Re}\left(\frac{\zeta_{0} q^{\prime \prime}\left(\zeta_{0}\right)}{q^{\prime}\left(\zeta_{0}\right)}+1\right)$.

Definition 2. [13, Definition 1] For $f \in A_{n}, n \in \mathbb{N}^{*}, m \in \mathbb{N}, \gamma \in \mathbb{C}$, let $I_{\gamma}$ be the integral operator given by $I_{\gamma}: A_{n} \rightarrow A_{n}$,

$$
\begin{gathered}
I_{\gamma}^{0} f(z)=f(z) \\
I_{\gamma}^{m} f(z)=\frac{\gamma+1}{z^{\gamma}} \int_{0}^{z} I_{\gamma}^{m-1} f(z) \cdot t^{\gamma-1} d t, z \in U .
\end{gathered}
$$

By using Definition 2, we can prove the following property for this integral operator:

For $f \in A_{n}, n \in \mathbb{N}^{*}, m \in \mathbb{N}, \gamma \in \mathbb{C}$, we have

$$
I_{\gamma}^{m} f(z)=z+\sum_{k=n+1}^{\infty}\left(\frac{\gamma+1}{\gamma+k}\right)^{m} a_{k} z^{k}, z \in U .
$$

Definition 3. [8, p. 4], [14, $p$. 36] Let $f$ and $F$ be analytic functions. The function $f$ is said to be subordinate to $F$, written $f \prec F$ or $f(z) \prec F(z)$, if there exists a function $w$ analytic in $U$, with $w(0)=0$ and $|w(z)|<1$, such that $f(z)=F(w(z))$. If $F$ is univalent, then $f \prec F$ if and only if $f(0)=F(0)$ and $f(U) \subset F(U)$. 
Definition 4. [14] A function $L(z, t), z \in U, t \geq 0$, is a subordination chain if $L(\cdot, t)$ is analytic and univalent in $U$ for all $t \geq 0$, and $L\left(z, t_{1}\right) \prec L\left(z, t_{2}\right)$, when $0 \leq t_{1}<t_{2}<\infty$.

Lemma B. 8, p. 4], [14, p. 159] The function

$$
L(z, t)=a_{1}(t) z+a_{2}(t) z^{2}+\ldots,
$$

with $a_{1}(t) \neq 0$ for $t \geq 0$ and $\lim _{t \rightarrow \infty}\left|a_{1}(t)\right|=\infty$ is a subordination chain if and only if there exist constant $r \in(0,1]$ and $M>0$ such that

(i) $L(z, t)$ is analytic in $|z|<r$ for each $t \geq 0$, locally absolutely continuous in $t \geq 0$ for each $|z|<r$, and satisfies

$$
|L(z, t)| \leq M\left|a_{1}(t)\right|, \text { for }|z|<r \text { and } t \geq 0 ;
$$

(ii) there exists a function $p(z, t)$ analytic in $U$ for all $t \in[0, \infty)$ and measurable in $[0, \infty)$ for each $z \in U$, such that $\operatorname{Re} p(z, t)>0$ for $z \in U, t \in[0, \infty)$ and

$$
\frac{\partial L(z, t)}{\partial t}=\frac{z \cdot \partial L(z, t)}{\partial z} \cdot p(z, t) \quad \text { or } \quad \operatorname{Re} \frac{z \cdot \partial L(z, t) / \partial z}{\partial L(z, t) / \partial t}>0, z \in U, t \geq 0
$$

for $|z|<r$ and for almost all $t \in[0, \infty)$.

Definition 5. [8, p. 9] The function $f \in \mathcal{H}(U)$ is called close-to-convex if there exists a starlike function $g$ such that

$$
\operatorname{Re} \frac{z f^{\prime}(z)}{g(z)}>0, z \in U
$$

In order to use the concept of fuzzy differential subordination, we remember the following definitions.

Definition 6. [5] A pair $\left(A, F_{A}\right)$, where $F_{A}: X \rightarrow[0,1]$ and

$$
A=\left\{x \in X ; 0 \leq F_{A}(x) \leq 1\right\}
$$

is called fuzzy subset of $X$. The set $A$ is called the support of the fuzzy set $\left(A, F_{A}\right)$ and $F_{A}$ is called the membership function of the fuzzy set $\left(A, F_{A}\right)$.

One can also denote $A=\operatorname{supp}\left(A, F_{A}\right)$.

If $A \subset X$, then

$$
F_{A}(x)=\left\{\begin{array}{lll}
1 & \text { if } & x \in A \\
0 & \text { if } & x \notin A .
\end{array}\right.
$$

A classical subset of $X$ can be considered as the fuzzy set of $X$, with the membership function $F_{A}$ defined as in (1).

Definition 7. $[9]$ Let $D \subset \mathbb{C}$ and let $z_{0} \in D$ be a fixed point. We take the functions $f, g \in \mathcal{H}(D)$. The function $f$ is said to be fuzzy subordinate to $g$ and we write $f \prec_{F} g$ or $f(z) \prec_{F} g(z)$, if there exists a function $F: \mathbb{C} \rightarrow[0,1]$, such that

(i) $f\left(z_{0}\right)=g\left(z_{0}\right)$;

(ii) $F(f(z)) \leq F(g(z))$ for all $z \in D$. 
Remark 8. Such function $F: \mathbb{C} \rightarrow[0,1]$ can be considered

$$
F(z)=\frac{|z|}{1+|z|}, F(z)=\frac{1}{1+|z|}, F(z)=|\sin | z||, F(z)=|\cos | z|| .
$$

Definition 9. [10] Let $\psi: \mathbb{C}^{3} \times \bar{U}-\mathbb{C}, a \in \mathbb{C}$, and let $h$ be univalent in $U$, with $h(0)=\psi(a, 0,0), q$ be univalent in $U$, with $q(0)=a$, and $p$ be analytic in $U$, with $p(0)=a$. Also, $\psi\left(p(z), z p^{\prime 2} p^{\prime \prime}(z) ; z\right)$ is analytic in $U$ and $F: \mathbb{C} \rightarrow[0,1]$.

If $p$ is analytic in $U$ and satisfies the (second-order) fuzzy differential subordination

$$
\psi\left(p(z), z p^{\prime 2} p^{\prime \prime}(z) ; z\right) \leq F(h(z)),
$$

i.e.

$$
\psi\left(p(z), z p^{\prime 2} p^{\prime \prime}(z) ; z\right) \prec_{F} h(z), z \in U,
$$

then $p$ is called a fuzzy solution of the fuzzy differential subordination. The univalent function $q$ is called a fuzzy dominant of the fuzzy solution of the fuzzy differential subordination, or more simple a fuzzy dominant, if

$$
p(z) \prec_{F} q(z), z \in U
$$

for all $p$ satisfying (2). A fuzzy dominant $\widetilde{q}$ that satisfies

$$
\widetilde{q}(z) \prec_{F} q(z), z \in U,
$$

for all fuzzy dominants $q$ of $(2)$ is said to be the fuzzy best dominant of $(2)$. Note that the fuzzy best dominant is unique up to a rotation in $U$.

Remark 10. The function $F: \mathbb{C} \rightarrow[0,1]$ can be a function of the form of functions shown in Remark 8 .

\section{MAin Results}

Theorem 11. Let $q$ be univalent in $U$ and let $\theta$ and $\phi$ be analytic functions in a domain $D$ containing $q(U)$, with $\phi(w) \neq 0$, when $w \in q(U)$.

Let

$$
F: U \rightarrow[0,1], F(z)=\frac{1}{1+|z|}, z \in U .
$$

Set

$$
Q(z)=z q^{\prime}(z) \cdot \phi[q(z)], h(z)=\theta[q(z)]+Q(z),
$$

and suppose that we have

(i) $Q$ is starlike;

(ii) $\operatorname{Re} \frac{z h^{\prime}(z)}{Q(z)}=\operatorname{Re}\left[\frac{\theta^{\prime}[q(z)]}{\phi[q(z)]}+\frac{z Q^{\prime}(z)}{Q(z)}\right]>0, z \in U$.

If $p$ is analytic in $U$, with $p(0)=q(0), p(U) \subset D$, then

$$
\frac{1}{1+\left|\theta[p(z)]+z p^{\prime}(z) \cdot \phi[p(z)]\right|} \leq \frac{1}{1+\left|\theta[q(z)]+z q^{\prime}(z) \cdot \phi[q(z)]\right|},
$$


that is

$$
F\left(\theta[p(z)]+z p^{\prime}(z) \cdot \phi[p(z)] \leq F\left(\theta[q(z)]+z q^{\prime}(z) \cdot \phi[q(z)]\right)\right.
$$

implies

$$
\frac{1}{1+|p(z)|} \leq \frac{1}{1+|q(z)|}, z \in U
$$

that is

$$
F(p(z)) \leq F(q(z)), z \in U
$$

and $q$ is the fuzzy best dominant, through $F$.

Proof. From (i) we know that the function $Q$ is starlike and from (ii) we know that the function $h$ is close-to-convex.

Let the function:

$$
\begin{aligned}
L(z, t) & =a_{1}(t) z+a_{2}(t) z^{2}+\ldots \\
& =h(z)+t Q(z)=\theta[q(z)]+(1+t) Q(z) \\
& =\theta[q(z)]+(1+t) z q^{\prime}(z) \cdot \phi[q(z)] .
\end{aligned}
$$

This function is analytic in $U$ for all $t \geq 0$ and is continuously differentiable on $[0, \infty)$ for $z \in U$.

Differentiating (5) with respect to $z$ we obtain

$$
\begin{aligned}
\frac{\partial L(z, t)}{\partial z} & =a_{1}(t)+2 a_{2}(t) z+\ldots \\
& =\theta^{\prime}[q(z)] q^{\prime}(z)+(1+t)\left\{q^{\prime}(z) \cdot \phi[q(z)]\right. \\
& \left.+z q^{\prime \prime}(z) \cdot \phi[q(z)]+z q^{\prime}(z) \cdot \phi^{\prime}[q(z)]\right\} .
\end{aligned}
$$

For $z=0$ we have

$$
\begin{aligned}
a_{1}(t) & =\theta^{\prime}[q(0)] \cdot q^{\prime}(0)+(1+t) q^{\prime}(0) \cdot \phi[q(0)] \\
& =q^{\prime}(0) \cdot \phi[q(0)] \cdot\left[\frac{\theta^{\prime}[q(0)]}{\phi[q(0)]}+1+t\right] \neq 0 .
\end{aligned}
$$

Differentiating (5) with respect to $t$ we obtain

$$
\frac{\partial L(z, t)}{\partial t}=Q(z)=z q^{\prime}(z) \cdot \phi[q(z)] .
$$

We calculate:

$$
\operatorname{Re} \frac{z \cdot \partial L(z, t) / \partial z}{\partial L(z, t) / \partial t}=\operatorname{Re}\left[\frac{\theta^{\prime}[q(z)]}{\phi[q(z)]}+(1+t) \frac{z Q^{\prime}(z)}{Q(z)}\right] .
$$

From (i) and (ii), $t \geq 0$, we have

$$
\operatorname{Re} \frac{z \cdot \partial L(z, t) / \partial z}{\partial L(z, t) / \partial t}=\operatorname{Re}\left[\frac{\theta^{\prime}[q(z)]}{\phi[q(z)]}+\frac{z Q^{\prime}(z)}{Q(z)}\right]+t \operatorname{Re} \frac{z Q^{\prime}(z)}{Q(z)}>0 .
$$


Hence $a_{1}(t) \neq 0, \lim _{t \rightarrow \infty}\left|a_{1}(t)\right|=\infty$ and

$$
\operatorname{Re} \frac{z \cdot \partial L(z, t) / \partial z}{\partial L(z, t) / \partial t}>0,
$$

for $z \in U$ and $t \geq 0$. Using Lemma $\mathrm{B}, L(z, t)$ is a subordination chain which by Definition 4 implies

$$
L(z, s) \prec L(z, t) \text { for } 0 \leq s \leq t .
$$

For $t=0$, (5) becomes $L(z, 0)=h(z)$, then (6) becomes

$$
h(z) \prec L(z, t), t \geq 0, z \in U .
$$

Using (7) and Definition 3, we have

$$
L(\zeta, t) \notin h(U),|\zeta|=1, t \geq 0 .
$$

Let the function $\psi: \mathbb{C}^{2} \times \bar{U} \rightarrow \mathbb{C}$,

$$
\psi(r, s)=\theta(r)+s \phi(r) .
$$

For $r=p(z), s=z p^{\prime}(z), z \in U$, we have

$$
\psi\left(p(z), z p^{\prime}(z)\right)=\theta[p(z)]+z p^{\prime}(z) \cdot \phi[p(z)]
$$

which is an analytic function since $\theta, \phi$ and $p$ are analytic functions.

For $r=q(z), s=z q^{\prime}(z)$, we have

$$
\psi\left(q(z), z q^{\prime}(z)\right)=\theta[q(z)]+z q^{\prime}(z) \cdot \phi[q(z)], z \in U .
$$

Then the fuzzy differential subordination (3) becomes

$$
\frac{1}{1+\left|\psi\left(p(z), z p^{\prime}(z)\right)\right|} \leq \frac{1}{1+\left|\psi\left(q(z), z q^{\prime}(z)\right)\right|}, \forall z \in \bar{U} .
$$

In order to prove that (3) or (9) implies $p$ is subordinate to function $q$, we apply Lemma A. For that we assume that the functions $p, q$ and $h$ satisfy the conditions in Lemma A in the unit disc $\bar{U}$.

Assume that function $p$ is not subordinate to function $q$.

By Lemma A, there exist points $z_{0}=r_{0} e^{i \theta_{0}} \in U$ and $\zeta_{0} \in \partial U \backslash E(q)$, and $m \geq n \geq 1$, that satisfy

$$
p\left(z_{0}\right)=q\left(\zeta_{0}\right), z_{0} p^{\prime}\left(z_{0}\right)=m \zeta_{0} q^{\prime}\left(\zeta_{0}\right) .
$$

Then

$$
\begin{aligned}
\psi\left(p\left(z_{0}\right), z_{0} p^{\prime}\left(z_{0}\right)\right) & =\theta\left[p\left(z_{0}\right)\right]+z_{0} p^{\prime}\left(z_{0}\right) \cdot \phi\left[p\left(z_{0}\right)\right] \\
& =\theta\left[q\left(\zeta_{0}\right)\right]+m \zeta_{0} q^{\prime}\left(\zeta_{0}\right) \cdot \phi\left[q\left(\zeta_{0}\right)\right] .
\end{aligned}
$$

If in (5) we take $t=m-1 \geq 0$, then

$$
L(z, m-1)=\theta[q(z)]+m Q(z)=\theta[q(z)]+m z q^{\prime}(z) \cdot \phi[q(z)] .
$$

For $z=\zeta_{0} \in \partial U \backslash E(q)$, (11) becomes

$$
L\left(\zeta_{0}, m-1\right)=\theta\left[q\left(\zeta_{0}\right)\right]+m \zeta_{0} q^{\prime}\left(\zeta_{0}\right) \cdot \phi\left[q\left(\zeta_{0}\right)\right],\left|\zeta_{0}\right|=1 .
$$


Using 10 and 12 , we have

$$
\psi\left(p\left(z_{0}\right), z_{0} p^{\prime}\left(z_{0}\right)\right)=L\left(\zeta_{0}, m-1\right), z_{0} \in U, \zeta_{0} \in \partial U \backslash E(q) .
$$

From (8), relation $(13)$ is equivalent to

$$
\frac{1}{1+\left|\psi\left(p\left(z_{0}\right), z_{0} p\left(z_{0}\right)\right)\right|} \geq \frac{1}{1+\left|\psi q\left(\zeta_{0}\right), m \zeta_{0} q^{\prime}\left(\zeta_{0}\right)\right|} .
$$

Relation (14) contradicts (9), which proves that the assumption we made is false, hence $p$ is subordinate to $q$, meaning

$$
\frac{1}{1+|p(z)|} \leq \frac{1}{1+|q(z)|} .
$$

Since $q$ is the solution of the univalent equation

$$
\theta[q(z)]+z q^{\prime}(z) \phi[q(z)]=h(z),
$$

we have that $q$ is the best dominant.

Theorem 12. Let $q$ be univalent in $U$, with $q(0)=1, q(z) \neq 0, z \in U$, and let $\theta: \mathbb{C} \rightarrow \mathbb{C}, \theta(w)=w$ and $\phi: \mathbb{C} \rightarrow \mathbb{C}, \phi(w)=\frac{1}{w}, \phi(w) \neq 0, w \neq 0$. Let

$$
F: U \rightarrow[0,1], F(z)=\frac{1}{1+|z|}, z \in U .
$$

Set

$$
\begin{gathered}
Q(z)=z q^{\prime}(z) \cdot \phi[q(z)] \\
h(z)=\theta[p(z)]+Q(z)=\theta[p(z)]+z q^{\prime}(z) \cdot \phi[q(z)]
\end{gathered}
$$

and suppose that we have

(j) $\operatorname{Re} \frac{\theta^{\prime}[q(z)]}{\phi[q(z)]}>0$;

(jj) $\operatorname{Re}\left[1+\frac{z q^{\prime \prime}(z)}{q^{\prime}(z)}-\frac{z q^{\prime}(z)}{q(z)}\right]>0, z \in U$.

For $m \in \mathbb{N}^{*}, \gamma \in \mathbb{C}$, the function $R$ is analytic in $U$,

$$
R(z)=\frac{z\left[I_{\gamma}^{m} f(z)\right]^{\prime \prime}}{\left[I_{\gamma}^{m} f(z)\right]^{\prime}}+\frac{z\left[I_{\gamma}^{m} f(z)\right]^{\prime}}{I_{\gamma}^{m} f(z)}-1+\frac{\left[I_{\gamma}^{m} f(z)\right]^{\prime} \cdot I_{\gamma}^{m} f(z)}{z} \neq 0, \quad z \in U .
$$

Then

$$
\begin{gathered}
\frac{1}{1+\left|\frac{z\left[I_{\gamma}^{m} f(z)\right]^{\prime \prime}}{\left[I_{\gamma}^{m} f(z)\right]^{\prime}}+\frac{z\left[I_{\gamma}^{m} f(z)\right]^{\prime}}{I_{\gamma}^{m} f(z)}-1+\frac{\left[I_{\gamma}^{m} f(z)\right]^{\prime} \cdot I_{\gamma}^{m} f(z)}{z}\right|} \\
\leq \frac{1}{1+\left|\theta[q(z)]+z q^{\prime}(z) \phi[q(z)]\right|},
\end{gathered}
$$

that is

$$
F(R(z)) \leq F(h(z))
$$


implies

$$
\frac{1}{1+\left|\frac{\left[I_{\gamma}^{m} f(z)\right]^{\prime} \cdot I_{\gamma}^{m} f(z)}{z}\right|} \leq \frac{1}{1+|q(z)|}, z \in U,
$$

that is

$$
F(p(z)) \leq F(q(z)), \quad z \in U
$$

and $q$ is the best dominant.

Proof. We let

$$
p(z)=\frac{\left[I_{\gamma}^{m} f(z)\right]^{\prime} \cdot I_{\gamma}^{m} f(z)}{z}, z \in U .
$$

Using Property 1, in (16) we have

$$
\begin{aligned}
p(z) & =\frac{\left[z+\sum_{k=n+1}^{\infty} \frac{(\gamma+1)^{m}}{(\gamma+k)^{m}} a_{k} z^{k}\right]^{\prime}\left[z+\sum_{k=n+1}^{\infty} \frac{(\gamma+1)^{m}}{(\gamma+k)^{m}} z_{k} z^{k}\right]}{z} \\
& =\frac{\left[1+\sum_{k=n+1}^{\infty} \frac{(\gamma+1)^{m}}{(\gamma+k)^{m}} a_{k} z^{k-1} k\right] \cdot z \cdot\left[1+\sum_{k=n+1}^{\infty} \frac{(\gamma+1)^{m}}{(\gamma+k)^{m}} a_{k} z^{k-1}\right]}{z} \\
& =\left(1+\sum_{k=n+1}^{\infty} \frac{(\gamma+1)^{m}}{(\gamma+k)^{m}} a_{k} \cdot k \cdot z^{k-1}\right)\left(1+\sum_{k=n+1}^{\infty} \frac{(\gamma+1)^{m}}{(\gamma+k)^{m}} z_{k} z^{k-1}\right),
\end{aligned}
$$

and $p(0)=1$.

Differentiating (16) and after a short calculus, we obtain

$$
p(z)+\frac{z p^{\prime}(z)}{p(z)}=\frac{z\left[I_{\gamma}^{m} f(z)\right]^{\prime \prime}}{\left[I_{\gamma}^{m} f(z)\right]^{\prime}}+\frac{z\left[I_{\gamma}^{m} f(z)\right]^{\prime}}{I_{\gamma}^{m} f(z)}-1+\frac{\left[I_{\gamma}^{m} f(z)\right]^{\prime} \cdot I_{\gamma}^{m} f(z)}{z} .
$$

We let the function

$$
\psi: \mathbb{C}^{2} \times \bar{U} \rightarrow \mathbb{C}, \psi(r, s)=r+\frac{s}{r}
$$

For $r=p(z), s=z p^{\prime}(z)$, we obtain

$$
\psi\left(p(z), z p^{\prime}(z)\right)=p(z)+\frac{z p^{\prime}(z)}{p(z)}, z \in U .
$$

Using (18) in (17), we have

$$
\begin{aligned}
\psi\left(p(z), z p^{\prime}(z)\right) & =\frac{z\left[I_{\gamma}^{m} f(z)\right]^{\prime \prime}}{\left[I_{\gamma}^{m} f(z)\right]^{\prime}}+\frac{z\left[I_{\gamma}^{m} f(z)\right]^{\prime}}{I_{\gamma}^{m} f(z)}-1 \\
& +\frac{\left[I_{\gamma}^{m} f(z)\right]^{\prime} \cdot I_{\gamma}^{m} f(z)}{z} .
\end{aligned}
$$


Since $\theta(w)=w, \theta[q(z)]=q(z), \phi(w)=\frac{1}{w}, \phi[q(z)]=\frac{1}{q(z)}, q(z) \neq 0$, we have

$$
Q(z)=z q^{\prime}(z) \cdot \frac{1}{q(z)}
$$

and

$$
h(z)=\theta[q(z)]+Q(z)=q(z)+\frac{z q^{\prime}(z)}{q(z)}, z \in U .
$$

Using (18) and (21), relation (15) becomes

$$
\frac{1}{1+\left|p(z)+\frac{z p^{\prime}(z)}{p(z)}\right|} \leq \frac{1}{1+\left|q(z)+\frac{z q^{\prime}(z)}{q(z)}\right|}, z \in U .
$$

In order to prove Theorem 12 , we shall use Theorem 11 . For that, we show that the necessary conditions are satisfied. Differentiating 20 and after a short calculus, we have

$$
\frac{z Q^{\prime}(z)}{Q(z)}=1+\frac{z q^{\prime \prime}(z)}{q^{\prime}(z)}-\frac{z q^{\prime}(z)}{q(z)}, z \in U .
$$

Using (jj) in (23) we have

$$
\operatorname{Re} \frac{z Q^{\prime}(z)}{Q(z)}>0, z \in U,
$$

hence the function $Q$ is starlike.

Differentiating (21) and using (j) and (24), and after a short calculus, we obtain

$$
\begin{aligned}
\operatorname{Re} \frac{z h^{\prime}(z)}{Q(z)} & =\operatorname{Re}\left[\frac{z \phi^{\prime}[q(z)] \cdot q^{\prime}(z)}{z q^{\prime}(z) \cdot \phi[q(z)]}+\frac{z Q^{\prime}(z)}{Q(z)}\right] \\
& =\operatorname{Re} \frac{\theta^{\prime}[q(z)]}{\phi[q(z)]}+\operatorname{Re} \frac{z Q^{\prime}(z)}{Q(z)}>0, z \in U .
\end{aligned}
$$

Since $\theta(w)=w$ and $\phi(w)=\frac{1}{w}$, we obtain

$$
\theta[p(z)]+z p^{\prime}(z) \cdot \phi[p(z)]=p(z)+\frac{z p^{\prime}(z)}{p(z)},
$$

and

$$
\theta[q(z)]+z q^{\prime}(z) \cdot \phi[q(z)]=q(z)+\frac{z q^{\prime}(z)}{q(z)} .
$$

Using (25) and 26) in (22, it becomes

$$
\frac{1}{1+\left|\theta[p(z)]+z p^{\prime}(z) \phi[p(z)]\right|} \leq \frac{1}{1+\left|\theta[q(z)]+z q^{\prime}(z) \cdot \phi[q(z)]\right|} .
$$

Since the conditions from Theorem 11 are satisfied, by applying it, we obtain

$$
\frac{1}{1+|p(z)|} \leq \frac{1}{1+|q(z)|}
$$


i.e.

$$
\frac{1}{1+\left|\frac{\left[I_{\gamma}^{m} f(z)\right]^{\prime} \cdot I_{\gamma}^{m} f(z)}{z}\right|} \leq \frac{1}{1+|q(z)|}, z \in U .
$$

Since $q$ is the solution of the univalent equation

$$
h(z)=q(z)+\frac{z q^{\prime}(z)}{q(z)},
$$

we have $q$ is the best dominant of 15 .

\section{EXAMPle}

Let $q(z)=1+z$, be an univalent function in $U$, with $q(0)=1$ and let the functions $\theta: \mathbb{C} \rightarrow \mathbb{C}, \theta(w)=w$ and $\phi: \mathbb{C} \rightarrow \mathbb{C}, \phi(w)=\frac{1}{w}, w \neq 0, w \in q(U)$.

If $q(z)=w$, then

$$
\theta[q(z)]=q(z)=1+z, \phi[q(z)]=\frac{1}{q(z)}=\frac{1}{1+z}, z \in U .
$$

We calculate:

(a) $\operatorname{Re} \frac{\theta^{\prime}[q(z)]}{\phi[q(z)]}=\operatorname{Re} \frac{(1+z)^{\prime}}{\frac{1}{1+z}}=\operatorname{Re}(1+z)>0, z \in U$;

(b) $\operatorname{Re}\left(1+\frac{z q^{\prime \prime}(z)}{q^{\prime}(z)}-\frac{z q^{\prime}(z)}{q(z)}\right)=\operatorname{Re}\left(1-\frac{z}{1+z}\right)$

$$
=\operatorname{Re} \frac{1}{1+z}>0, z \in U
$$

(c) $h(z)=\theta[q(z)]+Q(z)=q(z)+\frac{z q^{\prime}(z)}{q(z)}=1+z+\frac{z}{1+z}, z \in U$;

(d) $p(0)=q(0)=1$.

For $f \in A, f(z)=z+\frac{4}{3} z^{2}$ and $m=1, \gamma=2$, we obtain

$$
\begin{aligned}
I_{2}^{1} f(z) & =I_{2}^{1}\left(z+\frac{4}{3} z^{2}\right)=\frac{\gamma+1}{z^{\gamma}} \int_{0}^{z}\left(t+\frac{4}{3} t^{2}\right) t d t \\
& =\frac{3}{z^{2}} \int_{0}^{z}\left(t^{2}+\frac{4}{3} t^{3}\right) d t=\frac{3}{z^{2}}\left(\frac{z^{3}}{3}+\frac{4}{3} \cdot \frac{z^{4}}{4}\right) \\
& =z+z^{2} .
\end{aligned}
$$

The function

$$
\begin{gathered}
\frac{z\left[I_{2}^{1} f(z)\right]^{\prime \prime}}{\left[I_{2}^{1} f(z)\right]^{\prime}}+\frac{z\left[I_{2}^{1} f(z)\right]^{\prime}}{I_{2}^{1} f(z)}-1+\frac{\left[I_{2}^{1} f(z)\right]^{\prime} \cdot I_{2}^{1} f(z)}{z} \\
=\frac{3 z+4 z^{2}+(1+2 z)^{2}(1+z)^{2}}{(1+z)(1+2 z)}
\end{gathered}
$$


is analytic and

$$
p(z)=\frac{I_{\gamma}^{1}[f(z)]^{\prime} I_{\gamma}^{1}[f(z)]}{z}=(1+z)(1+2 z)
$$

is also an analytic function.

From Theorem 12 , we have:

$$
\frac{1}{1+\left|\frac{3 z+4 z^{2}+(1+2 z)^{2}(1+z)^{2}}{(1+z)(1+2 z)}\right|} \leq \frac{1}{1+\left|1+z+\frac{z}{1+z}\right|},
$$

that is

$$
F\left(\frac{3 z+4 z^{2}+(1+2 z)^{2}(1+z)^{2}}{(1+z)(1+2 z)}\right) \leq F\left(1+z+\frac{z}{1+z}\right)
$$

implies

$$
\frac{1}{1+|(1+2 z)(1+z)|} \leq \frac{1}{1+|1+z|}, z \in U
$$

that is

$$
F((1+z)(1+2 z)) \leq F(1+z), z \in U
$$

Declaration of Competing Interests The author has no competing interests to declare.

\section{REFERENCES}

[1] Alb Lupaş, A., A note on special fuzzy differential subordinations using multiplier transformation and Ruschewehy derivative, J. Computational Analysis and Applications, 25 (6) (2018), 1116-1124,

[2] Alb Lupaş, A., Oros, Gh., On special fuzzy differential subordinations using Sălăgean and Ruscheweyh operators, Applied Mathematics and Computation, 261 (2015), 119-127.

[3] Haydar, E. A., On fuzzy differential subordination, Mathematica Moravica,19 (1) (2015), 123 $-129$.

[4] Ibrahim, R. W., On the Subordination and Super-Ordination Concepts with Applications, Journal of Computational and Theoretical Nanoscience, 14 (5) (2017), 2248 - 2254.

[5] Gal, S. G., Ban, A. I., Elements of Fuzzy Mathematics, Editura Universităţii din Oradea, 1996. (in Romanian)

[6] Miller, S. S., Mocanu, P. T., Second order differential inequalities in the complex plane, J. Math. Anal. Appl., 65 (1978), 298-305.

[7] Miller, S. S., Mocanu, P. T., Differential subordinations and univalent functions, Michigan Math. J., 28 (1981), 157-171.

[8] Miller, S. S., Mocanu, P. T., Differential Subordinations. Theory and Applications, Marcel Dekker, Inc., New York, Basel, 2000.

[9] Oros, G. I., Oros, Gh., The notion of subordination in fuzzy sets theory, General Mathematics, 19 (4) (2011), 97-103.

[10] Oros, G. I., Oros, Gh., Fuzzy differential subordination, Acta Universitatis Apulensis, 3 (2012), 55-64.

[11] Oros, G. I., Oros, Gh., Dominants and best dominants in fuzzy differential subordinations, Stud. Univ. Babeş-Bolyai Math., 57 (2) (2012), 239-248. 
[12] Oros G.I., Briot-Bouquet fuzzy differential subordination, Analele Universităţii Oradea, Fasc. Mathematica, 19 (2) (2012), 83-97.

[13] Oros, G. I., Oros, Gh., Diaconu R., Differential subordinations obtained with some new integral operators, J. Computational Analysis and Applications, 19 (5) (2015), 904-910.

[14] Pommerenke Ch., Univalent Functions, Vanderhoeck and Ruprecht, Gottingen, 1975.

[15] Wanas, A. K., Majeed, A. H., Fuzzy differential subordination properties of analytic functions involving generalized differential operator, Sci.Int.(Lahore), 30 (2) (2018), 297-302. 